\title{
Correction: Kim, H.; et al. Geospatial Assessment of the Post-Earthquake Hazard of the 2017 Pohang Earthquake Considering Seismic Site Effects. ISPRS Int. J. Geo-Inf. 2018, 7, 375
}

\author{
Han-Saem Kim ${ }^{1} \oplus$, Chang-Guk Sun ${ }^{2, *}$ and Hyung-Ik Cho ${ }^{1}$ \\ 1 Earthquake Research Center, Korea Institute of Geoscience and Mineral Resources, Daejeon 305-350, Korea; \\ adoogen@kigam.re.kr (H.-S.K.); hicho@kigam.re.kr (H.-I.C.) \\ 2 Geological Research Division, Korea Institute of Geoscience and Mineral Resources, Daejeon 305-350, Korea \\ * Correspondence: pungsun@kigam.re.kr; Tel.: +82-42-868-3265
}

Received: 21 January 2019; Accepted: 24 January 2019; Published: 29 January 2019

We have recently been made aware of errata and omissions in the introduction section for describing the seismological characteristics of the 2017 Pohang earthquake as stated in the title of this article, which was recently published in ISPRS Int. J. Geo-Inf. [1]. The seismological characteristics (epicenter, depth, magnitude) of 2017 Pohang Earthquake, which was a great matter for the Korean seismologist, have been researched by a seismologist. Thus, we thought that there is a high probability of citation related to the errata because unsuitable quotes induce the different conclusions for seismologist. Thus, we changed the errata to consider the officially published information by the Korean government and to avoid confusion with other results. The authors would like to apologize for any inconvenience caused to the readers by these changes.

This study established a post-earthquake survey framework for the rapid earthquake damage estimation, correlated with seismic site effects, in order to identify the influence of site-specific effects for 2017 Pohang Earthquake. Seismic zonation was determined on the basis of seismic site effect by classifying sites using the multivariate site classification system. We believe that our changes are to clarify the introduction of epicenters considering the officially published information by the Korean Meteorological Administration [2]. Thus, the minor changes of epicenter of mainshock (also aftershocks) have no material impact on the conclusions of our paper. The authors wish to make the following six corrections to this paper [1]:

\section{Change in the First Paragraph of Introduction}

The authors wish to supplement the citation, which was existing references (reference \#1 and \#2 in the changed manuscript), to clarify the reference of major events of 2017 Pohang Earthquake, as follows:

Original text: "The 2017 Pohang earthquake occurred on 15 November 2017, in Heunghae, Pohang in the North Gyeongsang Province in South Korea. [ ... ] The clusters of epicenters were located along an unknown branch of the fault system as well as distributed across the Heunghae basin. Although the degree of seismic amplification of the 2017 Pohang earthquake was lower than that of the 2016 Gyeongju earthquake, the Pohang earthquake damage was more severe as its epicenters were spatially concentrated on unconsolidated Quaternary sediments (alluvial fans and granite wash)."

to the correct version, as follows:

Revised text: “The 2017 Pohang earthquake occurred on 15 November 2017, in Heunghae, Pohang in the North Gyeongsang Province in South Korea [1]. [ . . . ] The clusters of epicenters were located along 
an unknown branch of the fault system as well as being distributed across the Heunghae basin [2]. Although the degree of seismic amplification of the 2017 Pohang earthquake was lower than that of the 2016 Gyeongju earthquake, the Pohang earthquake damage was more severe as its epicenters were spatially concentrated on unconsolidated Quaternary sediments (alluvial fans and granite wash) [2]."

\section{Change in Figure 1 of Introduction}

Figure 1 described the epicenters and fault-plane solutions of major events of the 2017 Pohang Earthquake, and also visualized the spatial distribution of all aftershock epicenter (until July 2017) without the correct reference. The epicenters and fault-plane solutions were uncertain and needed further research by the seismologist. Thus, the correct epicenter of major events $(1$ mainshock and 6 aftershocks) was revised as Figure 1 using the officially published information by the Korean Meteorological Administration (reference \#1 in the changed manuscript) [2]. Additionally, the high-resolution satellite image with shaded by terrain (DEM) was applied. The author wishes to make the following correction to this paper [1]. Due to the errata, replace:

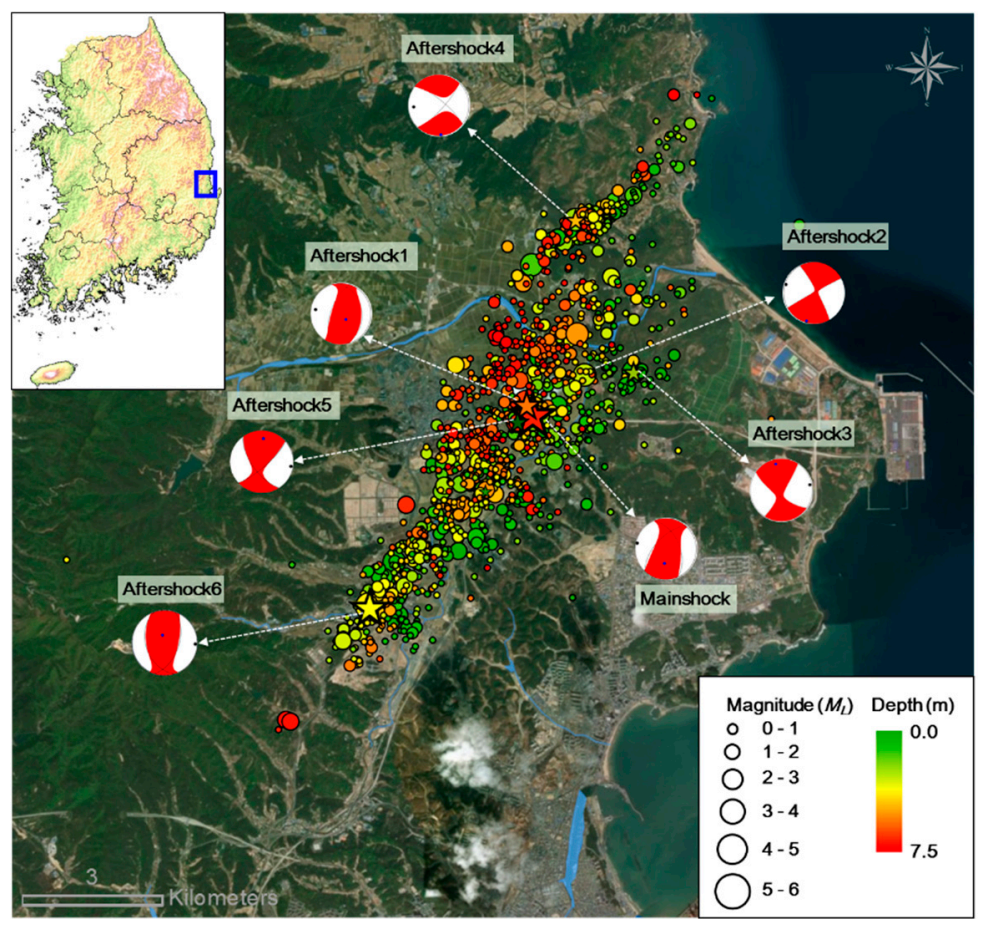

Figure 1. Epicenters and fault-plane solutions of the 2017 Pohang earthquake [2].

with 


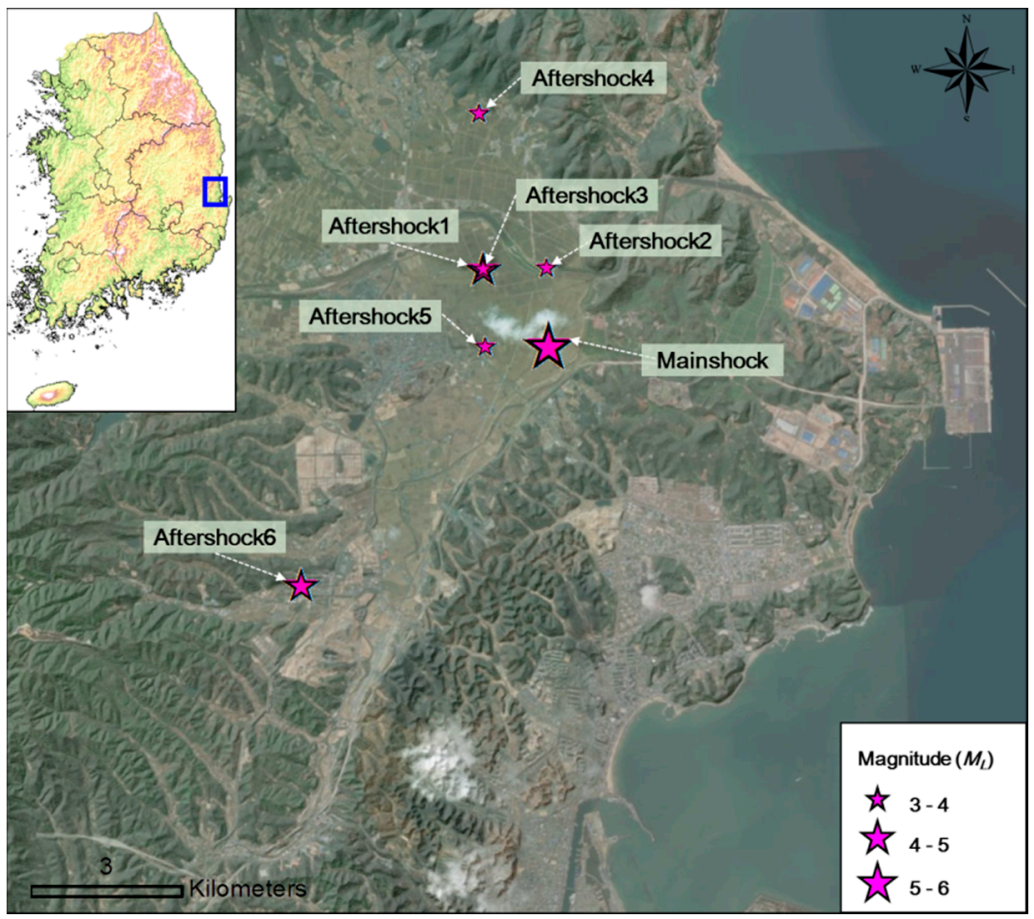

Figure 2. Epicenters of major events of the 2017 Pohang earthquake [1].

\section{Change in Table 1 of Introduction}

We have found the corresponding error in Table 1, likewise, in Figure 1. Table 1 and Figure 1 provide only the seismological information of major events of the 2017 Pohang Earthquake. To apply the correct time of occurrence, the coordinate of the epicenter, depth, magnitude $\left(M_{L}\right)$, Table 1 should be changed. The modification of Table 1 (Figure 1) was officially published information by the Korean Meteorological Administration (reference \#1 in the changed manuscript) [2].

Table 1. Major events of the 2017 Pohang earthquake [1].

\begin{tabular}{|c|c|c|c|c|c|c|}
\hline \multirow{2}{*}{ Earthquake } & \multirow{2}{*}{ Date and Time of Occurrence (KST) } & \multicolumn{2}{|c|}{ Epicenter } & \multirow{2}{*}{ Depth (km) } & \multicolumn{2}{|c|}{ Magnitude } \\
\hline & & Latitude & Longitude & & $M_{L}$ & $M_{w}$ \\
\hline Mainshock & 15 November 2017 14:29:32 & 36.1073 & 129.3686 & 4.0 & 5.5 & 5.4 \\
\hline Aftershock1 & 15 November 2017 16:49:31 & 36.1103 & 129.3647 & 5.5 & 4.6 & 4.3 \\
\hline Aftershock2 & 16 November 2017 09:02:43 & 36.1149 & 129.3851 & 2.0 & 3.8 & 3.6 \\
\hline Aftershock3 & 19 November 2017 23:45:48 & 36.1148 & 129.3770 & 3.9 & 3.9 & 3.5 \\
\hline Aftershock4 & 20 November 2017 06:05:16 & 36.1385 & 129.3748 & 3.7 & 3.8 & 3.6 \\
\hline Aftershock5 & 25 December 2017 16:19:23 & 36.1077 & 129.3629 & 5.6 & 3.8 & 3.5 \\
\hline Aftershock6 & 11 February 2018 05:03:04 & 36.0798 & 129.3340 & 4.3 & 4.8 & 4.6 \\
\hline
\end{tabular}

to the correct version, as follows: 
Table 2. Major events of the 2017 Pohang earthquake [1].

\begin{tabular}{|c|c|c|c|c|c|}
\hline \multirow{2}{*}{ Earthquake } & \multirow{2}{*}{ Date and Time of Occurrence (KST) } & \multicolumn{2}{|c|}{ Epicenter } & \multirow{2}{*}{ Depth (km) } & \multirow{2}{*}{$M_{L}$} \\
\hline & & Latitude & Longitude & & \\
\hline Mainshock & 2017-11-15 14:29:31 & 36.11 & 129.37 & 7.0 & 5.4 \\
\hline Aftershock1 & 2017-11-15 16:49:30 & 36.12 & 129.36 & 10.0 & 4.3 \\
\hline Aftershock2 & 2017-11-16 09:02:42 & 36.12 & 129.37 & 8.0 & 3.6 \\
\hline Aftershock3 & 2017-11-19 23:45:47 & 36.12 & 129.36 & 9.0 & 3.5 \\
\hline Aftershock4 & 2017-11-20 06:05:15 & 36.14 & 129.36 & 12.0 & 3.6 \\
\hline Aftershock5 & 2017-12-25 16:19:22 & 36.11 & 129.36 & 10.0 & 3.5 \\
\hline Aftershock6 & 2018-02-11 05:03:03 & 36.08 & 129.33 & 9.0 & 4.6 \\
\hline
\end{tabular}

\section{Change in Figure 3}

In Figure 3, the spatial distribution of all aftershock epicenters was also visualized. This modification corresponds with changes \#2 and \#3. Thus, the epicenters of seven major events were also modified. Additionally, five multi-layered geo-data were visualized on the same area to help to understand the spatial modeling of geo-data.

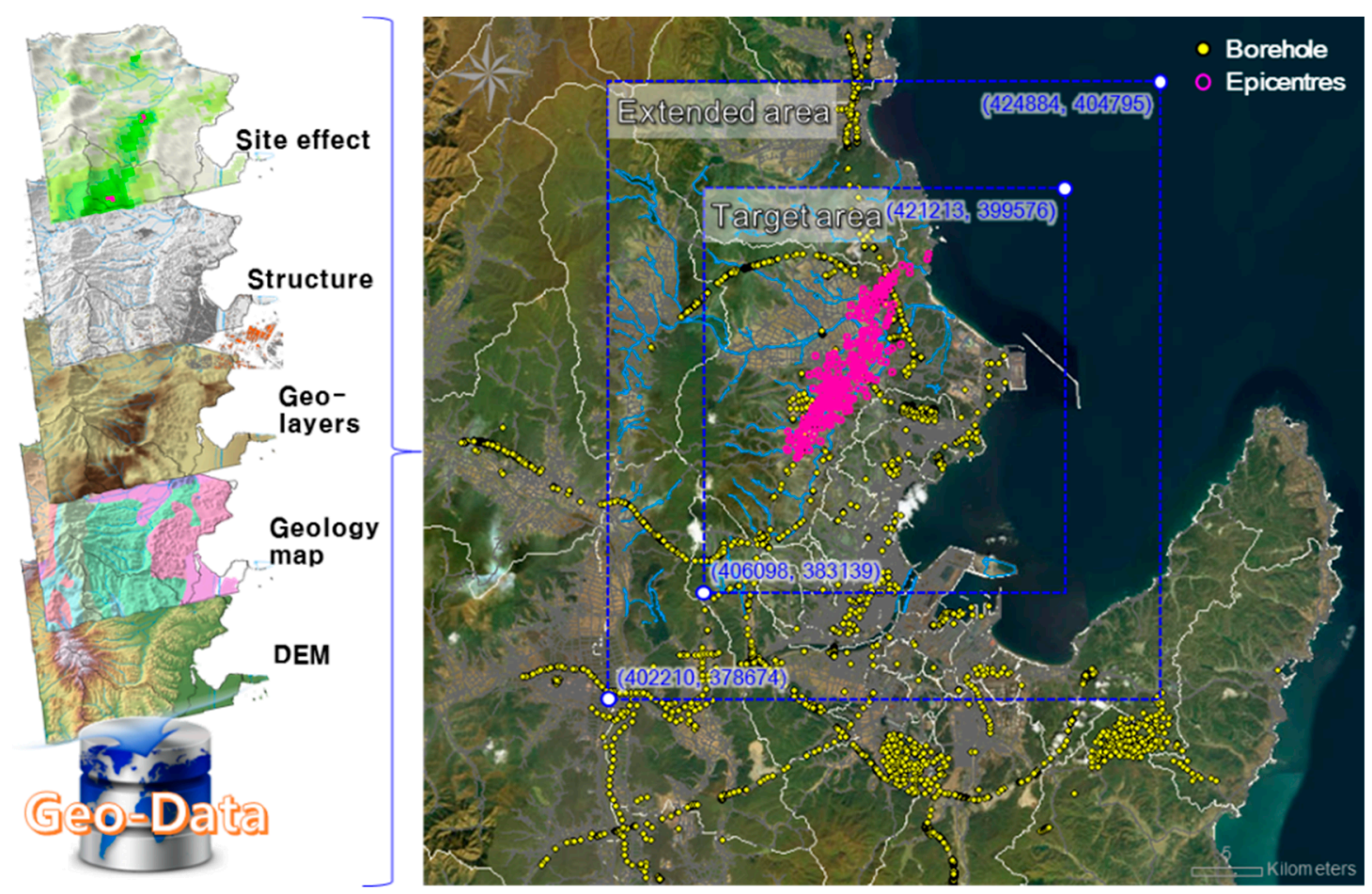

Figure 3. Extended and target areas for spatial modeling of geo-data in Pohang.

to the correct version, as follows: 


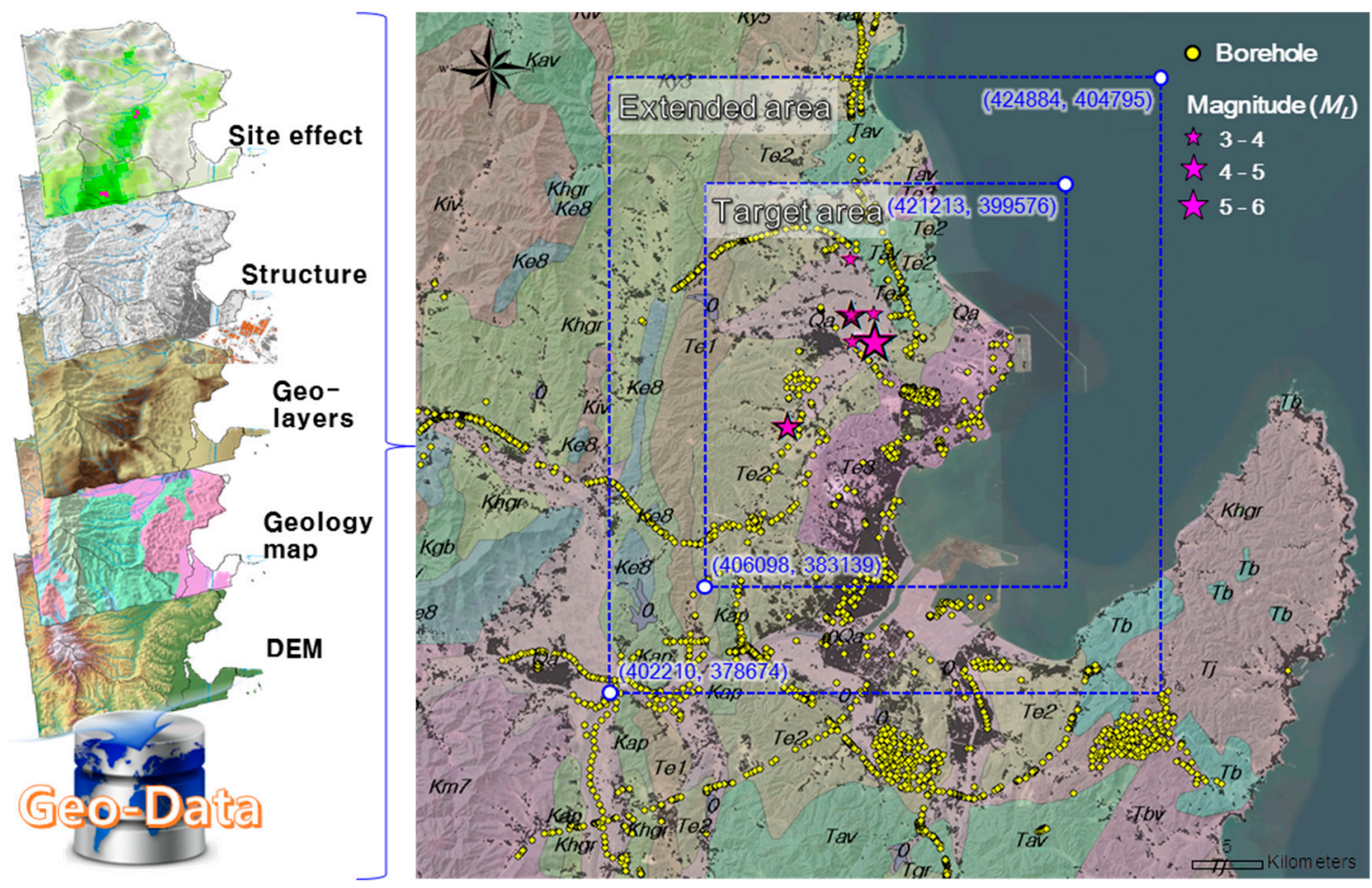

Figure 4. Extended and targeted areas for spatial modeling of geo-data in Pohang.

\section{Change in Figure 9}

Figure 9 shows the spatial comparison between $T_{G}$-based seismic zonation and earthquake damage category of buildings. Additionally, the spatial distribution of all aftershock epicenter was also visualized on the spatial comparison map to help reader to understand the epicenter. Thus, the correct epicenter of major events (corresponding changes to Figure 1 and Table 1) was also modified.
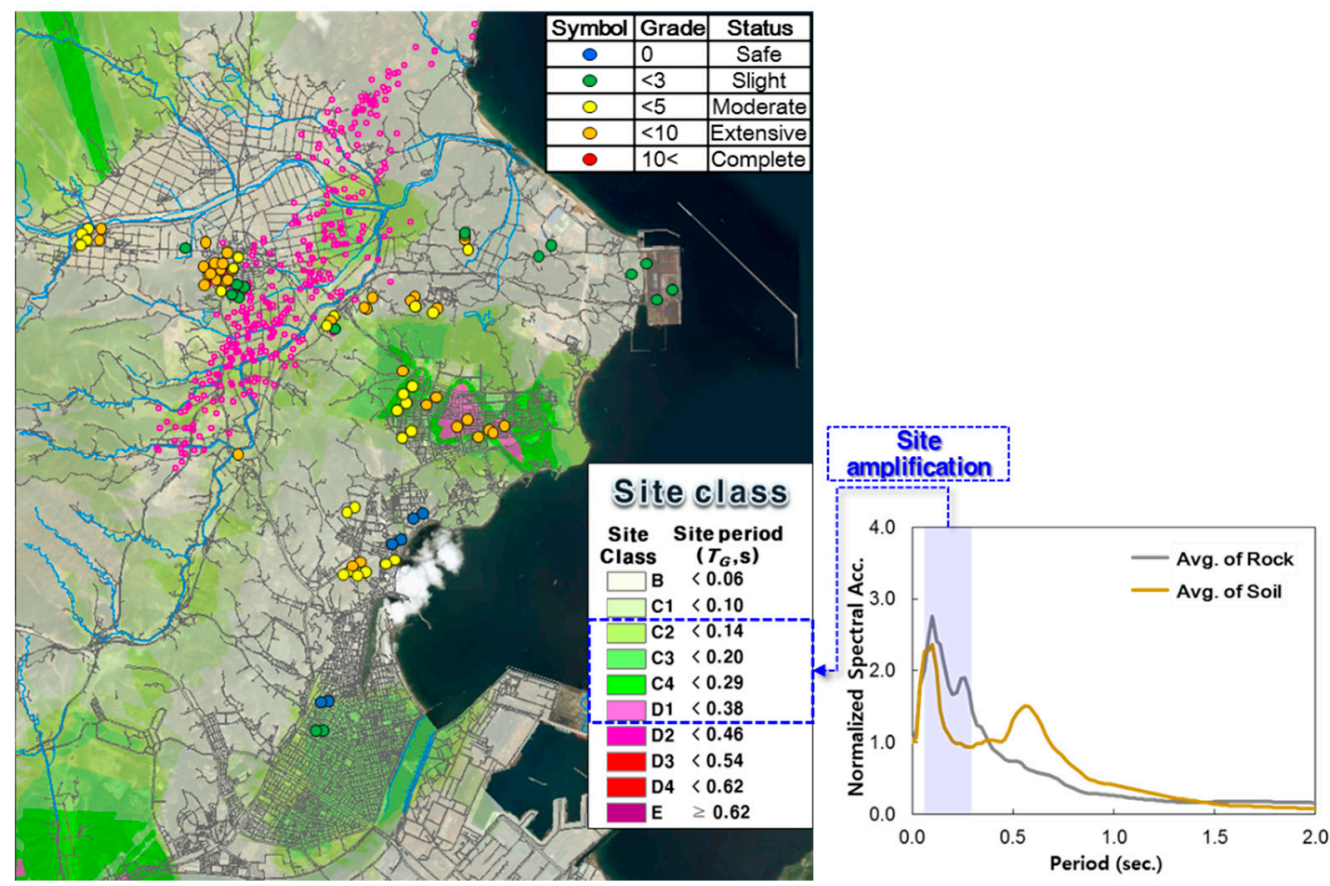

Figure 5. Spatial comparison between $T_{G}$-based seismic zonation and earthquake damage category of buildings. 
to the correct version, as follows:
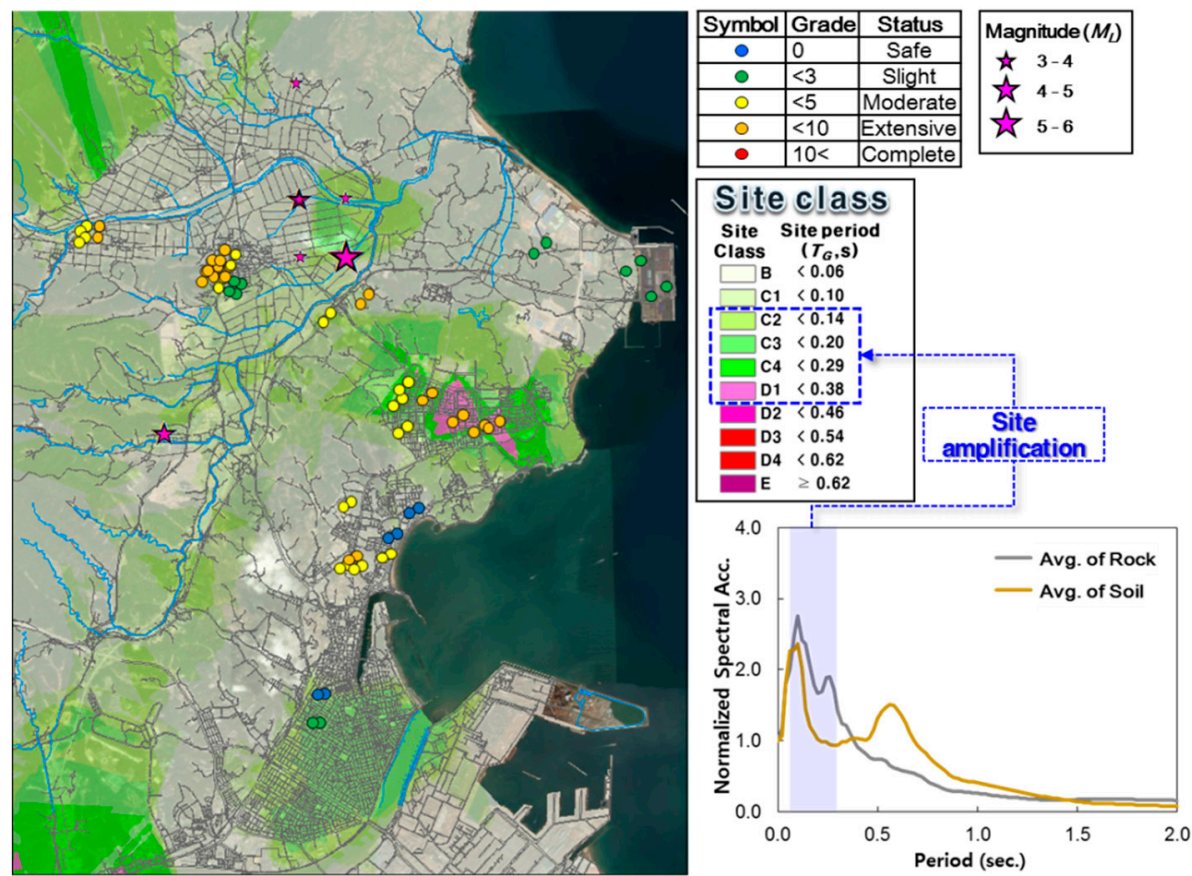

Figure 6. Spatial comparison between $T_{G}$-based seismic zonation and earthquake damage category of buildings.

\section{Change in References (Reference \#2)}

The accessed date of reference \#1 in the reference list should be changed to apply the up-to-date earthquake record. And reference \#2 in the reference list should be modified to clarify the correct English title, which is the Korean report (reference \#2 in the changed manuscript) [3].

\section{Original Reference \#1 and \#2:}

1. Korea Meteorological Administration (KMA). Earthquake: Notice, Korea Meteorological Administration. Available online: http://www.kma.go.kr/weather/earthquake_volcano/ (accessed on 17 April 2018).

2. Korea Institute of Geoscience and Mineral Resources (KIGAM). Brochure for Korean Peninsula Southeastern Earthquake; KIGAM: Daejeon, Korea, 2018.

to the correct version, as follows:

\section{Revised Reference \#1 and \#2:}

1. Korea Meteorological Administration (KMA). Earthquake: Notice, Korea Meteorological Administration. Available online: http://www.kma.go.kr/weather/earthquake_volcano/ (accessed on 28 January 2019).

2. Korea Institute of Geoscience and Mineral Resources (KIGAM). Earthquakes in the Southeast Korean Peninsula: Focusing on the 2016 Gyeongju and the 2017 Pohang Earthquakes; KIGAM: Daejeon, Korea, 2018. (In Korean) 


\section{References}

1. Kim, H.S.; Sun, C.G.; Cho, H.I. Geospatial assessment of the post-earthquake hazard of the 2017 Pohang earthquake considering seismic site effects. ISPRS Int. J. Geo-Inf. 2018, 7, 375. [CrossRef]

2. Korea Meteorological Administration (KMA). Earthquake: Notice, Korea Meteorological Administration. Available online: http:/ / www.kma.go.kr/weather/earthquake_volcano/ (accessed on 28 January 2019).

3. Korea Institute of Geoscience and Mineral Resources (KIGAM). Earthquakes in the Southeast Korean Peninsula: Focusing on the 2016 Gyeongju and the 2017 Pohang Earthquakes; KIGAM: Daejeon, Korea, 2018. (In Korean)

2019 by the authors. Licensee MDPI, Basel, Switzerland. This article is an open access article distributed under the terms and conditions of the Creative Commons Attribution (CC BY) license (http:// creativecommons.org/licenses/by/4.0/). 